\title{
THE CONJUGACY PROBLEM FOR FINITE GRAPH PRODUCTS
}

\author{
K. J. HORADAM
}

(Communicated by Warren J. Wong)

\begin{abstract}
A finite graph product is the fundamental group of a finite graph of groups. Finite graph products with finite cyclic edge groups are shown to inherit a solvable conjugacy problem from their vertex groups under certain conditions, of which the most important is that all the edge group generators in each vertex group are powers of a common central element.
\end{abstract}

\section{INTRODUCTION}

A solvable conjugacy problem (S.C.P.) is generally not inherited by graph products of groups with S.C.P., even in the simplest cases of HNN extensions and free products with amalgamation [2]. If all the edge groups are cyclic the problem is more tractable. In [1] the author conjectured that, under appropriate recursive conditions, if the graph is finite and all the edge group generators in each vertex group are powers of a common central element then the graph product has S.C.P. (This is not true in general if the graph is infinite [1, 3.7].)

This paper shows that a finite graph product with finite cyclic edge groups, which satisfies these conditions, has S.C.P.. The argument does not use the recursive set formulation of the conjugacy problem given in [1,3.3], but instead relies on induction on the number of edges not in a maximal tree of the graph. Notably, however, the proof is still carried out in the fundamental groupoid of the graph of groups, rather than the graph product, since use of both group and graph-theoretic techniques streamlines the inductive step. The reader is referred to [3] for the theory of computability and [4] for the theory of graph products.

\section{MACHINERY}

Notation is based on [1]. Let $(\mathscr{G}, D)$ be a fixed finite graph of groups with the following description. The nonempty finite connected directed graph $D=$ $(E, V)$ has known (and disjoint) edge set $E$ and vertex set $V$, and source

Received by the editors July 8, 1988 and, in revised form, October 6, 1988.

1980 Mathematics Subject Classification (1985 Revision). Primary 20F10, 20E06; Secondary $20 \mathrm{~L} 10$.

Key words and phrases. Graph product, fundamental group, graph of groups, conjugacy problem, HNN extension, free product with amalgamation. 
and terminus maps $s, t: E \rightarrow V$; respectively. For each $v$ in $V$, the vertex group $G_{v}$ has a recursive presentation. For each $e$ in $E$, the edge group $G_{e}$ is presented as a cyclic group on a single generator $k_{e}$. The two group monomorphisms $G_{e} \mapsto G_{t e}$ (given by $k_{e} \rightarrow k_{\rho e}$ ) and $G_{e} \mapsto G_{s e}$ (given by $k_{e} \rightarrow k_{\lambda e}$ ) are partially recursive for each $e$ in $E$. For each $v$ in $V, H_{v} \subseteq G_{v}$ is the (recursive) set

$$
H_{v}=\left\{\dot{k}_{\rho e}: t e=v\right\} \cup\left\{k_{\lambda e}: s e=v\right\} .
$$

Let $\mathscr{F}$ be the fundamental groupoid of $(\mathscr{G}, D)$ and $G^{*}$ its graph product (fundamental group). The required result for $\mathscr{F}$ is built up from that for an intermediate sequence of fundamental groupoids $\mathscr{F}_{i}$ whose definition follows.

Definition 2.1. Let $T$ be a maximal tree in $D$ with known edge set. The edges of $D$ not in $T$ will be labelled

$$
e_{1}, e_{2}, \ldots, e_{m}, \quad m \geq 0 .
$$

Define $D_{i}=\left(E_{i}, V\right), 0 \leq i \leq m$, recursively, by:

$$
\begin{aligned}
D_{0} & =T, \\
E_{i} & =E_{i-1} \cup\left\{e_{i}\right\}, \quad 1 \leq i \leq m,
\end{aligned}
$$

so that $D_{m}=D$. Let $\left(\mathscr{G}_{i}, D_{i}\right)$ be the graph of groups obtained by restricting $(\mathscr{G}, D)$ to $D_{i}$, and let $\mathscr{F}_{i}$ be the fundamental groupoid of $\left(\mathscr{G}_{i}, D_{i}\right), 0 \leq i \leq m$, so that $\mathscr{F}_{0}$ is the fundamental groupoid of the tree of groups $\left(\mathscr{G}_{0}, T\right)$; on identifying stable letters with edges, $\mathscr{F}_{i}$ has the presentation $\mathscr{F}_{i}=\left\langle\operatorname{gen} \mathscr{F}_{i-1}, e_{i}\right.$ : $\left.\operatorname{rel} \mathscr{F}_{i-1}, e_{i} k_{\rho e_{i}} e_{i}^{-1}=k_{\lambda e_{i}}\right\rangle, 1 \leq i \leq m ;$ and $\mathscr{F}_{m}=\mathscr{F}$.

By $[1,2.1], D_{i}$ is a recursive graph, and $\mathscr{F}_{i}$ is a recursively presented groupoid, and there is an algorithm to decide whether or not an arbitrary element of $\mathscr{F}_{i}$ is a loop (and thus to distinguish elements of $G^{*}$ in $\mathscr{F}$ ).

The following conditions are imposed on $(\mathscr{G}, D)$.

\section{Conditions 2.2.}

(i) $G_{e}$ is finite, $\forall e \in E$,

(ii) $G_{v}$ has S.C.P., $\forall v \in V$,

(iii) there exists (known) $c_{v}: H_{v} \subseteq\left\langle c_{v}\right\rangle \subseteq \zeta\left(G_{v}\right)$, the centre of $G_{v}, \forall v \in V$, and

(iv) $\left\langle c_{v}\right\rangle$ has solvable extended word problem in $G_{v}, \forall v \in V$.

These conditions imply that the order of $c_{v}$ and the power $k_{\rho e}$ is of $c_{t e}$ and $k_{\lambda e}$ is of $c_{s e}$ can all be calculated. For $e=e_{i}, 1 \leq i \leq m$, define $p_{i}$ and $q_{i}$ by

$$
k_{\lambda e}=c_{s e}^{p_{i}} \text { and } k_{\rho e}=c_{t e}^{q_{i}}
$$




\section{The CONJUGACY PROBLEM}

It is proved in $[1,3.1]$ that the conjugacy problem is solvable for the set of pairs of elements of $\mathscr{F}$ (and hence of $G^{*}$ ) for which at least one element of the pair has nonzero reduced length, so that the question remaining is whether or not there is an algorithm to decide if any two elements of $\mathscr{F}$ with zero reduced length are conjugate.

Theorem 3.1. Under conditions (2.2), $\mathscr{F}$, and hence $G^{*}$, has S.C.P..

Proof. (by induction). (a) $\mathscr{F}$ has S.C.P. since $D_{0}$ is a tree, by $[1,3.5]$. Assume that $\mathscr{F}_{i-1}$ has S.C.P. for elements of zero reduced length, for some $i \geq 1$.

(b) Proof that $\mathscr{F}_{i}$ has S.C.P. for elements of zero reduced length. By [1, p. 347] it is sufficient to consider words $g \in\left\langle c_{v}\right\rangle$ and $h \in\left\langle c_{w}\right\rangle$ in $\mathscr{F}_{i}$, with $o(g)=o(h)$. Since $V$ is finite and, for each $w \in V$, the order of every power of $c_{w}$ is known, the candidate set

$$
C=\left\{c_{w}^{p}: w \in V, 1 \leq p<o\left(c_{w}\right), o\left(c_{w}^{p}\right)=o(g)\right\}
$$

of potential conjugates of $g$ in $\mathscr{F}_{i}$ (indeed, in $\mathscr{F}$ ) is known and finite.

By the inductive hypothesis, the set $A$ of all ordered pairs of elements in $C$ conjugate in $\mathscr{F}_{i-1}$ is known, and is an equivalence relation on $C$.

Consider edge $e_{i}$ in $\mathscr{F}_{i}$. Write $s e_{i}=s, t e_{i}=t, C_{s}=C \cap\left\langle c_{s}\right\rangle$ and $C_{t}=$ $C \cap\left\langle c_{t}\right\rangle$. Then $c_{s}^{\alpha} \in C_{s}$ and $c_{t}^{\beta} \in C_{t}$ satisfy $c_{t}^{\beta}=e_{i}^{-1} c_{s}^{\alpha} e_{i}$ if and only if $[1$, p. 347] there exists an integer $r$ such that

$$
\alpha \equiv p_{i} r \quad \bmod o\left(c_{s}\right), \quad \beta \equiv q_{i} r \quad \bmod o\left(c_{t}\right) .
$$

Hence the set $B$ of all ordered pairs of elements in $C$ conjugate by either $e_{i}$ or $e_{i}^{-1}$ (or, if $s=t$, by a power of loop $e_{i}$ ) is finite and can be found.

Clearly (c.f. $(* *)$ of $\left[1\right.$, p. 347]), $g$ is conjugate to $h$ in $\mathscr{F}_{i}$ if and only if there is a finite sequence

$$
g=c_{1}, c_{2}, \ldots, c_{p}=h
$$

such that the pairs $\left(c_{j-1}, c_{j}\right)$ are in $A$ and $B$ alternately as $j$ runs from 2 to $p$ (starting in either $A$ or $B$ ).

An algorithm to find all conjugates of $g$ in $\mathscr{F}_{i}$ would thus generate all such sequences of length $p$ starting with $g$ and containing no repetitions, for each $p \geq 1$. The algorithm can be terminated at $p=|C|$ since at most $|C|$ distinct conjugates of $g$ exist.

(c) By induction $\mathscr{F}$ has S.C.P. for elements of zero reduced length. Consequently $\mathscr{F}$ and $G^{*}$ have S.C.P..

This answers in the affirmative the conjecture of [1] in the case that the edge groups of $(\mathscr{G}, D)$ are all finite (possibly trivial) cyclic groups. In fact, more has been proved. If Condition 2.2(i) is removed, the subsequent comments still apply, on restricting the proof of (3.1) to elements of finite order, since if $G_{e_{i}}$ is infinite cyclic, then $C_{s}=C_{t}=B=\varnothing$, and element $g$ has the same set of conjugates in $\mathscr{F}_{i}$ as in $\mathscr{F}_{i-1}$. 
Corollary 3.2. Under conditions $2.2(\mathrm{ii})-(\mathrm{iv}), \mathscr{F}$, and hence $G^{*}$, has S.C.P. for elements of finite order.

The order criterion effectively separates the conjecture into two parts: the one answered here and the case where all the cyclic edge groups are infinite. This latter case remains unresolved (even for an HNN extension with two or more stable letters $[1,3.6])$ and is restated here.

Conjecture. Under conditions (2.2) with $2.2(\mathrm{i})$ replaced by $2.2(\mathrm{i})^{*} G_{e}$ is infinite, $\forall e \in E$, then $\mathscr{F}$, and hence $G^{*}$, has S.C.P.

\section{REFERENCES}

1. K. J. Horadam, The conjugacy problem for graph products with central cyclic edge groups, Proc. Amer. Math. Soc. 91 (1984), 345-350.

2. C. F. Miller III, On group theoretic decision problems and their classification, Ann. of Math. Stud., no. 68, Princeton Univ. Press, Princeton, N.J., 1971.

3. H. Rogers, Jr. Theory of recursive functions and effective computability, McGraw-Hill, New York, 1968.

4. J.-P. Serre, Trees (translated by J. Stillwell), Springer-Verlag, Berlin, 1980.

Department of Mathematics, Royal Melbourne institute of Technology, Melbourne, Victoria, Australia, 3000. 